\title{
Rapid Melting and Quenching with Microsecond Current Pulses*
}

\author{
BRUCE M. CLEMENS \\ Physics Department, General Motors Research Laboratories, Warren, MI $48090-9055$ (U.S.A.) \\ R. M. GILGENBACH \\ Nuclear Engineering Department, The University of Michigan, Ann Arbor, MI 48109 (U.S.A.)
}

\begin{abstract}
We report the use of microsecond current pulses to transform layered crystalline $\mathrm{Ni}-\mathrm{Zr}$ films to amorphous alloys. The starting materials were electron-beam-or sputter-deposited multilayers with a composition modulation wavelength of $34 \mathrm{~nm}$, an average composition of $\mathrm{Ni}_{63} \mathrm{Zr}_{37}$ and a total thickness of $680 \mathrm{~nm}$. The electrical pulses were approximately rectangular and about $3 \mathrm{~ms}$ in duration, with an intensity of several hundred ampères, directly coupling 1.6-3 J of energy uniformly into the film. By monitoring current and voltage, the reaction and melting of the sample were observed, and the total energy of the pulse was easily computed. A sharp threshold in pulse energy for sample transformation was observed. A simple heat flow calculation demonstrated that the chemical energy released by the reaction and the change in diffusion kinetics as the sample temperature exceeded the glass transition temperature of the amorphous alloy are responsible for this sudden onset. The maximum temperature estimated from this calculation is below the melting point of the constituents, and the cooling rate is $10^{7}-10^{8} \mathrm{~K}^{-1}$ which is in agreement with the formation of amorphous alloys.
\end{abstract}

\section{Introduction}

The series of International Conferences on Rapidly Quenched Metals is among the indications that there is much interest and activity in the production of metastable phases by rapid quenching. High cooling rates $\left(10^{6} \mathrm{~K} \mathrm{~s}^{-1}\right)$ can be achieved by impinging molten material onto a cool heat sink, as in melt spinning or splat cooling. Alternatively, the surface region of a solid can be melted by rapid local heating, resulting in rapid quenching as heat flows from the heated surface region to the non-heated bulk. The latter method has been performed with laser, electron and pulsed ion beam heating, and cooling rates as high as

*Paper presented at the Sixth International Conference on Rapidly Quenched Metals, Montréal, August 3-7, 1987.
$10^{12} \mathrm{~K} \mathrm{~s}^{-1}$ have been claimed [1]. We recently introduced microsecond current pulses as a technique for rapid surface heating and have used this technique to produce amorphous alloys [2]. A high current density (about $10^{7} \mathrm{~A} \mathrm{~cm}^{-2}$ ), in the form of rectangular pulses of a few microseconds' duration, produces reaction and melting in multilayer $\mathrm{Ni}-\mathrm{Zr}$ films. The use of an insulating substrate (sapphire or oxidized silicon) confines the power input to the metal film, resulting in sharp thermal gradients and rapid cooling after the pulse.

Electrical pulses have several attractive features for rapid thermal treatment of metal films. Since the power is applied only to the conductive layer, the heated region is well defined and adjustable, with a sharp interface between the heated and non-heated regions. This is opposed to conventional surface-melting techniques where the heated depth is determined by the exponential attenuation of the radiation employed. The energy is coupled directly to the film (no reflection) and is deposited uniformly through the depth of the film. Energy deposition is also uniform across the area of the film so that large areas can be treated. The pulse shape can be manipulated to give the desired temporal behavior over several orders of magnitude. By monitoring the voltage and current, the sample resistance can easily be monitored; so phase changes can be directly observed, and energy deposited can be easily computed. This provides this method with a built-in diagnostic unavailable with other techniques.

The $\mathrm{Ni}-\mathrm{Zr}$ system was chosen for this study for a variety of reasons. The amorphous phase readily forms by conventional liquid quenching over a wide composition range, and its properties have been extensively studied [3]. Solid state reaction during longtime anneals of multilayered $\mathrm{Ni}-\mathrm{Zr}$ films also results in amorphous phase formation [4], and investigations of this process on a fast time scale are of fundamental interest in understanding the role of nucleation of the competing phases. There is a large negative heat of mixing between the constituents and so an atomic 
scale multilayered structure contains substantial chemical energy which can be released by reactions on the microsecond time scale. The effect of this chemical energy is to produce a self-sustained reaction which is triggered by the microsecond current pulses.

\section{Experiment}

$\mathrm{Ni}-\mathrm{Zr}$ multilayer samples were prepared by rotating the sapphire substrates above shielded electron beam evaporation sources in a vacuum system with a base pressure below $10^{-8}$ Torr, or by a similar method in a dual-source magnetron deposition system with a base pressure in the $10^{-8}$ Torr range. The rates were feedback controlled and set to produce an average composition of $\mathrm{Ni}_{63} \mathrm{Zr}_{37}$; the rotary motion was set to produce a composition modulation wavelength of $34 \mathrm{~nm} .20$ bilayers were deposited, resulting in a total thickness of $680 \mathrm{~nm}$. X-ray diffraction was performed in thin film Seeman-Bohlin geometry which increased the diffraction intensity from the surface layer over that observed in conventional scattering geometry. The sample orientation and the incident angle were chosen to eliminate Bragg peaks from the single-crystal substrate. The radiation employed was $\mathrm{Cu} K \alpha$. Auger depth profiling was performed on selected samples before and after pulsing to determine the degree of mixing and the level of impurities.

Samples were heated by a pulsed discharge circuit consisting of a multistage artificial transmission line. After the circuit has been charged to the desired voltage $(4-10 \mathrm{kV})$, the output pulse was extracted through a triggered spark gap switch. A $1.9 \Omega$ resistor was connected in series between the pulse generator and the sample. A low inductance $8 \Omega$ resistor connected in parallel with the sample served two purposes. First, measurement of the current through the parallel resistor gave the sample voltage. Second, the parallel resistor diverted some of the sample current after the sample resistance increased at the melt transition. The currents in the sample and parallel resistor were inductively measured with current transformers. The pulse had a $1.5 \mathrm{~ms}$ flat top with a rise time of about $0.75 \mathrm{~ms}$. The flat top in the current pulse resulted in negligible inductive contribution to the measurement of sample voltage. In order to distribute the pulsed current uniformly, the ends of the sample were pressed onto copper tape electrodes on a glass plate. The glass plate and sample were mounted in a low inductance holder which plugged into the discharge circuit. The samples were pulsed in air or in argon at atmospheric pressure. Pre-discharge and postdischarge resistance were measured with the sample in the holder.

\section{Results}

The structure of the starting material was that of the polycrystalline elements, as shown by the X-ray diffraction in Fig. 1(a). Clearly evident are peaks from f.c.c. nickel and h.c.p. zirconium. Pulses with an energy of $2.3 \mathrm{~J}$ or greater resulted in amorphous phase formation. Figure 1(b) shows X-ray diffraction for a sample pulsed with $2.3 \mathrm{~J}$ electrical energy input. The nickel and zirconium crystalline peaks are no longer evident, and a broad band characteristic of amorphous material has appeared. There is also a weak peak which can be attributed to zirconium oxide, which Auger depth profiling shows to be confined to the top $15 \mathrm{~nm}$ of the surface region. Auger depth profiling (Fig. 2) also showed that the initial strong composition modulation was completely destroyed by the pulse. The surface of the sample both before and after pulsing is mostly smooth and featureless as seen by scanning electron microscopy. However, after pulsing, there are some pinholes with smooth rounded edges.

The reaction of the layers can be clearly observed in the current and voltage traces during the pulse. Shown in Fig. 3 are the current and voltage traces for

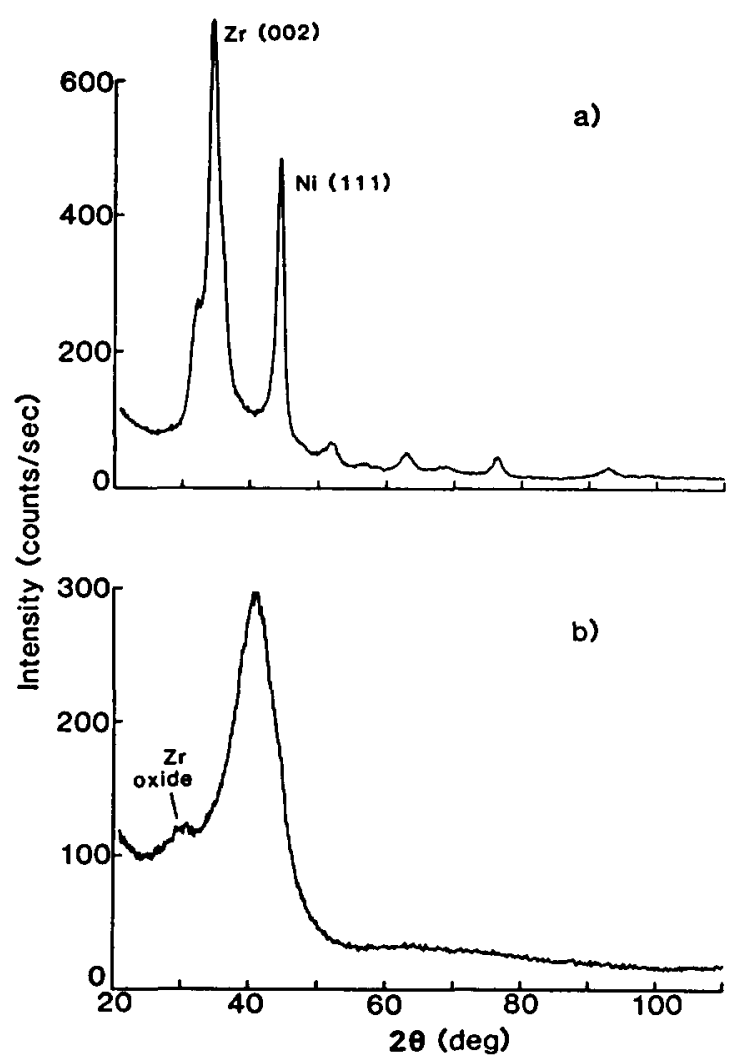

Fig. 1. Seeman-Bohlin X-ray diffraction for $\mathrm{Ni}-\mathrm{Zr}$ samples (a) before and (b) after a $2.3 \mathrm{~J}$ current pulse. 

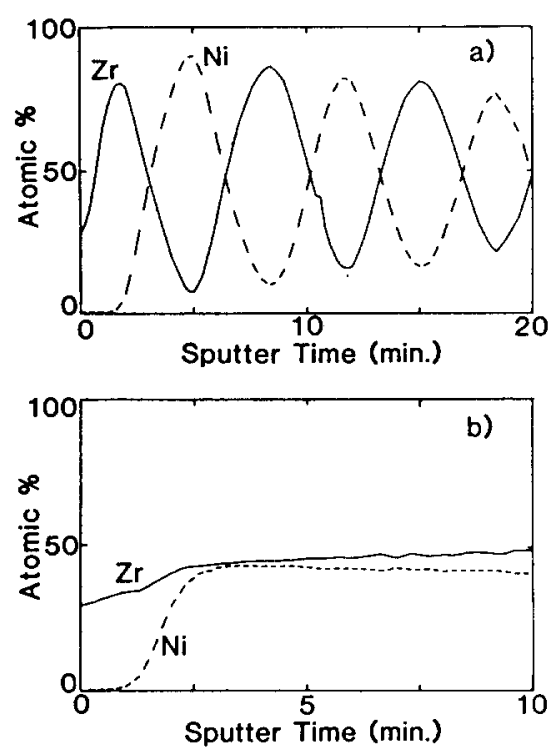

Fig. 2. Auger depth profiles of (a) an as-deposited and (b) a current-pulsed sample.

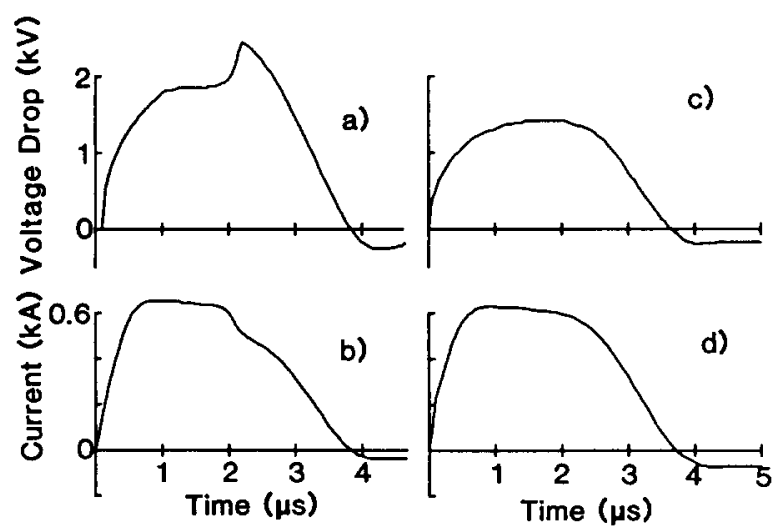

Fig. 3. Voltage and current traces for current pulses: (a) voltage for a $2.9 \mathrm{~J}$ pulse; (b) current for a $2.9 \mathrm{~J}$ pulse; (c) voltage for a $2.0 \mathrm{~J}$ pulse; (d) current for a $2.0 \mathrm{~J}$ pulse.

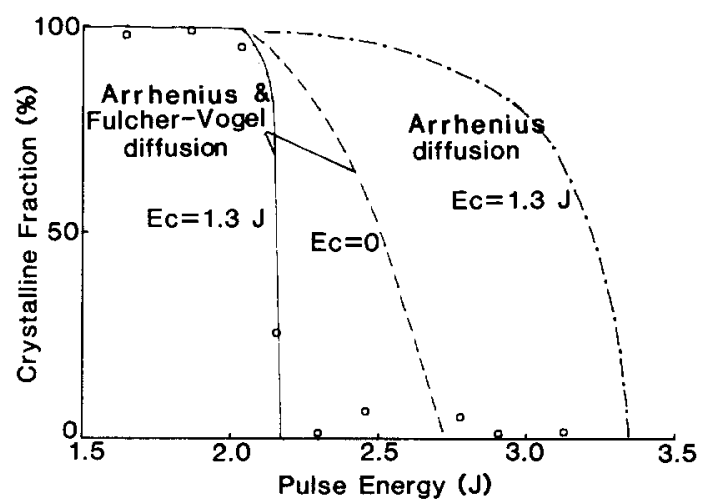

Fig. 4. Volume fraction of the sample remaining crystalline after pulse $v s$. pulse energy: $\bigcirc$, derived from experimental $\mathrm{X}$ ray data; _ , results of a model which includes a chemical driving term and both Arrhenius and Fulcher-Vogel diffusivity. pulses of 2.0 and $2.9 \mathrm{~J}$. The rapid rise in sample resistance evident in the traces from the $2.9 \mathrm{~J}$ pulse (Figs. $3(a)$ and 3(b)) is typical of samples where amorphous phase formation is observed. Pulses with an energy of $2 \mathrm{~J}$ or less produced no transition, as shown in Figs. 3(c) and 3(d).

The fraction of crystalline material remaining after the sample had been pulsed was calculated from the integrated X-ray diffraction intensity associated with crystalline peaks. Figure 4 shows this fraction plotted $v s$. pulse energy. A sharp drop occurs between 2 and $2.3 \mathrm{~J}$, which is where the resistive transition begins to appear. An increase of $15 \%$ in pulse power in going from 2 to $2.3 \mathrm{~J}$ results in a decrease in crystalline peak intensity from $97 \%$ to $4 \%$.

\section{Discussion}

Several physical phenomena are occurring as the sample heats during the pulse. Extrapolation of low temperature data [4] reveals that solid state formation of an amorphous alloy will begin to occur on the microsecond time scale at a temperature of about $1000 \mathrm{~K}$, well below the melting point of the constituents. This reaction will release the substantial heat of mixing which will cause a further temperature increase, resulting in a self-sustained reaction. Furthermore the diffusion kinetics in the reacted amorphous product will change in nature as the glass transition temperature $T_{\mathrm{g}}$ of the amorphous phase is exceeded. Diffusion in the solid state follows an Arrhenius form while diffusivity in liquid metals follows the Fulcher-Vogel form:

$D=D_{0} \exp \left(-\frac{B}{T-T_{0}}\right)$

In equilibrium, the cross-over between these two regimes will occur at $T_{\mathrm{g}}$, which for amorphous $\mathrm{Ni}-\mathrm{Zr}$ depends on composition and is between 600 and $850 \mathrm{~K}$ [3]. In conditions of rapid heating, the crossover temperature will occur when the atomic jump frequency becomes comparable with the time scale of the experiment [5]. Using the diffusivity from low temperature solid state reaction studies [4], we find a cross-over temperature of $900 \mathrm{~K}$.

In order to gain insight into the temporal behavior of the sample temperature during these pulses, and to understand the effects of the exothermic reaction between the constituents and the change in diffusion kinetics above $T_{\mathrm{g}}$, a simple heat flow calculation was performed. We used the Green function solution to the heat diffusion equation to find temperature as a 
function of position and time:

$$
\begin{aligned}
T(x, t)= & \int_{0}^{t} \int_{-\infty}^{+\infty} \frac{1}{\left\{4 \pi a^{2}(t-\tau)\right\}^{1 / 2}} \\
& \times \exp \left\{\frac{-(x-\xi)^{2}}{4 a^{2}(t-\tau)}\right\} f(\xi, \tau) \mathrm{d} \xi \mathrm{d} \tau
\end{aligned}
$$

where $a$ is the thermal diffusivity (taken to be that of the substrate) and $f(x, t)$ is the thermal driving function, which is the power (both electrical and chemical) divided by the heat capacity of the film. The magnitude of the chemical energy term was calculated by the method of Miedema [6] to be $1.3 \mathrm{~J}$, which is a substantial fraction of the electrical pulse energy of about $2 \mathrm{~J}$. The rate of chemical energy release is proportional to the growth rate of the interfacial alloy. This was calculated using the layer growth law

$d=(4 D t)^{1 / 2}$

For temperatures below the diffusion cross-over temperature, the diffusivity $D$ followed an Arrhenius form with a pre-factor and activation energy taken from solid state reaction data [4]. Above the crossover temperature the Fulcher-Vogel form (eqn. (1)) was used for the diffusivity, with rough estimates for the parameters selected in a manner similar to that used by Spaepen and Lin [7]. The thermal diffusivity of the substrate was used as an adjustable parameter so that the model calculation produced reaction at the observed pulse input energy. The value found in this manner $\left(0.036 \mathrm{~cm}^{2} \mathrm{~s}^{-1}\right)$ is in good agreement with published data for this temperature range [8]. Further details of this model have been previously published [2].

For pulses with energy greater than $2.3 \mathrm{~J}$, this model predicts a sharp rise in sample temperature because of the influence of chemical energy. This is reminiscent of the sharp rise in sample resistance observed in these pulses (Fig. 3). This sharp rise is not observed in either the model or the sample resistance with lower pulse energies. It is also not observed in the model calculation without incorporating the chemical term. The self-sustained reaction produces a sharp threshold in pulse energy for transformation as shown in Fig. 4, where the fraction remaining crystalline is plotted $v s$. pulse energy for the model calculation both with and without the chemical driving term. Also shown are the results of the model for the case where the diffusivity follows an Arrhenius form throughout the temperature range, and we see that the sharp threshold in pulse energy for transformation is not observed. The reaction temperatures predicted by this model are around $1000 \mathrm{~K}$; so this is not, strictly speaking, a solid state reaction since the amorphous phase is above $T_{\mathrm{g}}$. Since the reaction temperatures predicted are below the melting point of the $\mathrm{Zr}-\mathrm{Ni}$ crystalline phases [3], the transformation is taking place with the product in an undercooled liquid state. An estimate of the cooling rate can be calculated from this model by dividing the temperature drop from the melting point to $T_{\mathrm{g}}$ by the time that it took the sample to cool over this range. This is about $10^{7}-10^{8} \mathrm{~K} \mathrm{~s}^{-1}$, which is in good agreement with the observation of amorphous phase formation. The heating rate found from this calculation is about $4 \times 10^{8} \mathrm{~K} \mathrm{~s}^{-1}$.

\section{Conclusions}

Electric current pulses offer the ability to treat films thermally on a microsecond time scale. This technique has several advantages over conventional techniques including a built-in diagnostic which allows calculation of deposited energy and sample resistance. We have used this technique to produce amorphous $\mathrm{Ni}-$ $\mathrm{Zr}$ from layered crystalline film. A simple model calculation demonstrates the role of chemical energy which in this system induces a self-sustained reaction. The transformation from layered crystalline elements to amorphous alloy does not involve melting of the constituents but occurs above the glass transition temperature of the amorphous alloy and below the melting point of the crystalline phases.

\section{Acknowledgments}

We would like to acknowledge helpful conversations with W. L. Johnson and F. Spaepen. Auger depth profiling was performed by A. Dow and S. Simko of the Analytical Chemistry Department of General Motors Research. S. Bidwell and M. Devour performed some of the sample preparation.

\section{References}

1 C.-J. Lin, F. Spaepen and D. Turnbull, J. Non. Cryst. Solids, $61-62$ (1984) 767.

2 B. M. Clemens, R. M. Gilgenbach and S. Bidwell, Appl. Phys. Lett., 50 (1987) 495.

3 Z. Altounian, T. Guo-hua and J. O. Strom-Olsen, J. Appl. Phys., 54 (1983) 3111.

4 B. M. Clemens, Phys. Rev. B, 33 (11) (1986) 7615.

5 F. Spaepen, personal communication, 1986.

6 A. R. Miedema, Philips Tech. Rev., 36(1976) 217.

7 F. Spaepen and C. J. Lin, in M. von Allmen (ed.), Amorphous Metals and Non-equilibrium Processing, Materials Research Society Symp. Proc., Les Editions de Physique, Les Ulis, 1984 , p. 65

8 Y. S. Touloukian and C. Y. Ho (eds.), Thermophysical Properties of Matter, IFI-Plenum, New York, 1970, Vol. 2, p. 93; Vol. 5, pp. 24, 25 